\title{
Burnett Coefficients in Quantum Many-Body Systems
}

\author{
R. Steinigeweg ${ }^{1,2}$ \\ ${ }^{1}$ Institute for Theoretical Physics, Technical University Braunschweig, D-38106 Braunschweig, Germany \\ ${ }^{2}$ J. Stefan Institute, SI-1000 Ljubljana, Slovenia \\ T. $\operatorname{Prosen}^{3}$ \\ ${ }^{3}$ Faculty of Mathematics and Physics, University of Ljubljana, SI-1000 Ljubljana, Slovenia
}

(Dated: August 15, 2018)

\begin{abstract}
The Burnett coefficient $B$ is investigated for transport in one-dimensional quantum many-body systems. Extensive numerical computations in spin-1/2 chains suggest a linear growth with time, $B(t) \sim t$, for non-integrable chains exhibiting diffusive transport. For integrable spin chains in the metallic regime, on the other hand, we find a cubic growth with time, $B(t) \sim-D_{\mathrm{m}}^{2} t^{3}$, with the proportionality constant being simply a square of the Drude weight $D_{\mathrm{m}}$. The results are corroborated with additional studies in non-interacting quantum chains and in the classical limit of large-spin chains.
\end{abstract}

PACS numbers: 05.60.Gg, 05.70.Ln, 75.10.Pq

Introduction.- Understanding classical and quantum diffusion in deterministic Hamiltonian systems is one of the most ubiquitous problems of statistical physics [1]. In Fourier space of momentum $q$, diffusion is described by the well-known equation

$$
\dot{\rho}_{q}(t)=-q^{2} D(t) \rho_{q}(t), \quad D(t)=D
$$

and manifests as the simple exponential relaxation of a harmonic density profile at a characteristic time scale $\tau=1 / q^{2} D$. The strict derivation of diffusion from truly microscopic principles remains a challenge to theorists [2], and the problem is often simplified to a mere calculation of the diffusion coefficient $D(t)$ in the limit $q=0$ via the famous Green-Kubo formula [3]. It has become clear that $D(t)$ can diverge in integrable systems [4], $D(t) \propto t$ [5], due to the lack of sufficient scattering, which is a key issue for understanding large thermal spin transport in quantum magnets [6] or thermalization in cold atomic gases [7]. On the other hand, $D(t)$ is believed to be constant, $D(t)=D$, in generic nonintegrable systems as a consequence of microscopic Hamiltonian chaos [1]. This believe opens the important question whether diffusion is rather the rule than the exception.

The existence of diffusion can only be clarified by taking into account finite momentum $q \neq 0[8,9]$. The first higher order correction can be systematically described by the so-called Burnett coefficient $B(t)[1,10,11]$,

$$
\dot{\rho}_{q}(t)=\left[-q^{2} D(t)+q^{4} B(t)+\ldots\right] \rho_{q}(t),
$$

which may diverge even for dynamical processes with a constant $D(t)[10$. Even though Burnett coefficients have been extensively studied in the literature for various classical models, in particular for Lorentz-type gases [11, 12], essentially nothing is known about Burnett coefficients in quantum systems.

In this Letter we do the first steps by calculating $B(t)$ numerically for various one-dimensional, integrable and

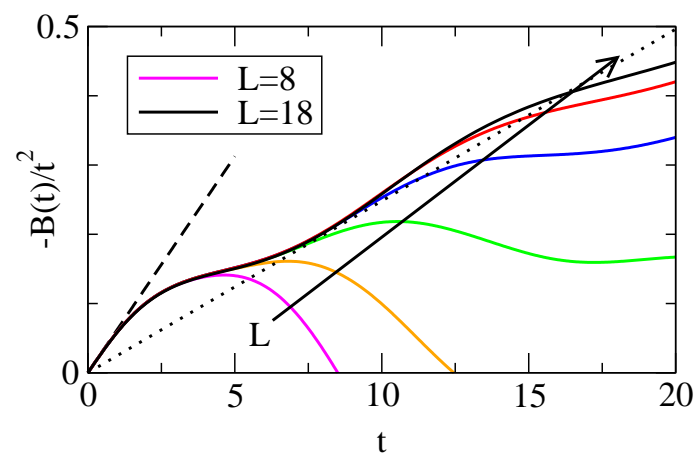

FIG. 1. (color online) Burnett coefficient $B(t)$, divided by $t^{2}$, for the spin- $1 / 2 X X Z$ chain at $\Delta=0.5$ for $L=8,10, \ldots, 18$ and all magnetization sectors. At short times or at $\Delta=0$ (free fermions), $B(t) / t^{2}$ is given by the function $-t / 16$ (dashed curve). In addition, a function $-(0.63)^{2} t / 16$ is indicated (dotted curve), see prediction (10).

nonintegrable models, including spin- $1 / 2 X X Z$ chains, large-spin chains, and more abstract models of quantum transport. We generally observe the moderate divergence $B(t) \sim B^{\prime} t$, for cases with a constant $D(t) \sim D$. At the characteristic time scale $\tau$, this observation implies

$$
\dot{\rho}_{q}(t=\tau)=\left[-q^{2} D+q^{2} B^{\prime} / D+\ldots\right] \rho_{q}(t=\tau),
$$

i.e., Burnett coefficients are a relevant correction at any finite momentum $q \neq 0$, which speed up or slow down the still diffusive relaxation (if $\left|B^{\prime}\right| \lesssim D^{2}$ ). For ballistic cases with $D(t) \sim D_{\mathrm{m}} t$, on the other hand, we find the stronger divergence $B(t) \sim-D_{\mathrm{m}}^{2} t^{3}$ with the Drude weight $D_{\mathrm{m}}$ as the constant of proportionality.

Diffusion and Burnett coefficient.- Following [10, 12, 13] the quantum Burnett coefficient $B(t)$ may be introduced by formally expanding the decay rate of densitydensity correlation functions in $q$. This expansion leads 
to the time-dependent diffusion coefficient

$$
D(t)=\frac{1}{\chi} \int_{0}^{t} \mathrm{~d} t_{1} f\left(t_{1}\right), \quad f\left(t_{1}\right)=\left\langle J(0) J\left(t_{1}\right)\right\rangle,
$$

given as a time integral over the two-point correlation function of the current operator $J(t)$ in the Heisenberg picture, where $\langle\bullet\rangle=\operatorname{tr}(\bullet) /$ dim denotes an equilibrium expectation at high temperatures, as considered in this Letter. The further occurring prefactor $\chi$ is a constant and denotes the "static susceptibility" [14].

The time-dependent Burnett coefficient is the difference between two contributions,

$$
B(t)=I_{4}(t)-I_{2-2}(t),
$$

where the first term $I_{4}(t)$ is given by

$$
I_{4}(t)=\frac{2}{\chi} \int_{0}^{t} \mathrm{~d} t_{1} \int_{0}^{t_{1}} \mathrm{~d} t_{2} \int_{0}^{t_{2}} \mathrm{~d} t_{3}\left\langle J(0) J\left(t_{1}\right) J\left(t_{2}\right) J\left(t_{3}\right)\right\rangle
$$

as a triple-time integral over the time-ordered four-point current autocorrelation function. The second term

$$
\begin{aligned}
I_{2-2}(t) & =\frac{2}{\chi} \int_{0}^{t} \mathrm{~d} t_{1} \int_{0}^{t_{1}} \mathrm{~d} t_{2} \int_{0}^{t_{2}} \mathrm{~d} t_{3}\left[f\left(t_{1}\right) f\left(t_{2}-t_{3}\right)\right. \\
& \left.+f\left(t_{2}\right) f\left(t_{1}-t_{3}\right)+f\left(t_{3}\right) f\left(t_{1}-t_{2}\right)\right]
\end{aligned}
$$

is a similar time-ordered integral but over products of two-point correlations [15]. Conveniently, this contribution can be rewritten as $I_{2-2}(t)=2 \chi D(t) \int_{0}^{t} \mathrm{~d} t_{1} D\left(t_{1}\right)$, particularly unveiling the linear increase $I_{2-2}(t) \propto t$ in the case of a existent diffusion constant. But, despite the apparent divergence of $I_{2-2}(t)$ in that case, the Burnett coefficient can still remain finite, as discussed in the following. To this end, assume for the moment that (i) the two-point correlation $f(t)$ is a delta function $\delta(t)$ and that (ii) the four-point correlation $\left\langle J(0) J\left(t_{1}\right) J\left(t_{2}\right) J\left(t_{3}\right)\right\rangle$ can be factorized as $f\left(t_{3}\right) f\left(t_{1}-t_{2}\right)$. Then the contributions $I_{2-2}(t)$ and $I_{4}(t)$ are identical and, as a consequence, the Burnett coefficient vanishes exactly. Giving up the assumption (i) by broadening the delta function still allows for a finite Burnett coefficient. While the assumption (ii) appears to be crucial, it may be fulfilled for a non-integrable model with $J$, in the energy eigenbasis, being a random Hermitian matrix and, consequently, $J^{2}$ being close to proportional to an identity matrix.

Anisotropic spin-1/2 Heisenberg chain.- We are going to investigate the transport of magnetization in the spin-1/2 $X X Z$ model as a paradigmatic example of an interacting many-particle quantum system in one dimension. The $X X Z$ Hamiltonian is given by

$$
H=\sum_{r=1}^{L}\left(S_{r}^{x} S_{r+1}^{x}+S_{r}^{y} S_{r+1}^{y}+\Delta S_{r}^{z} S_{r+1}^{z}\right),
$$
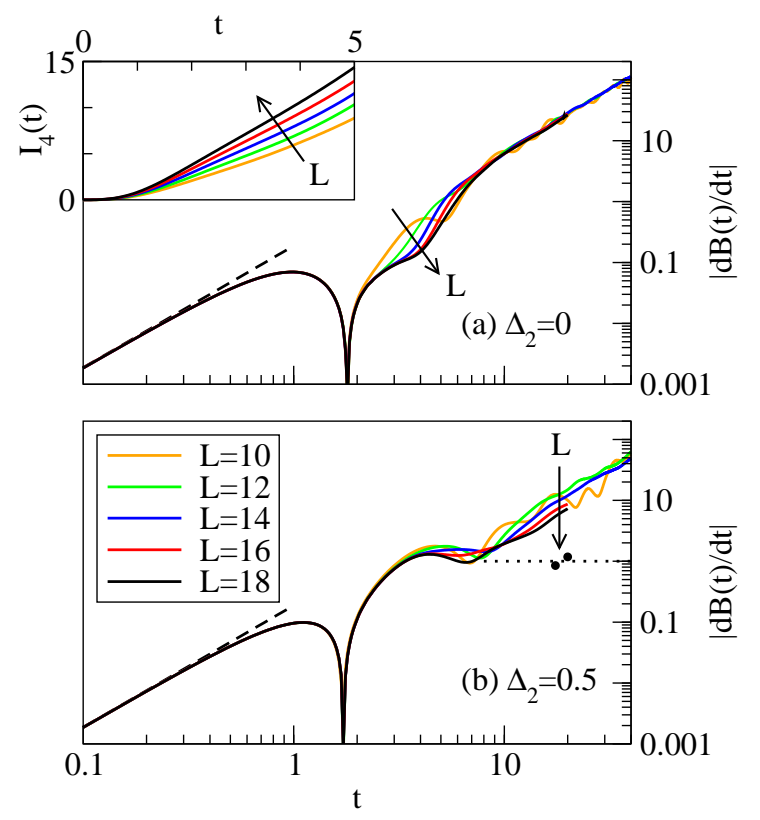

FIG. 2. (color online) Absolute value of the derivated Burnett coefficient, $|\mathrm{d} B(t) / \mathrm{d} t|$, for the spin- $1 / 2 X X Z$ chain at $\Delta=1.5$ (a), and for its non-integrable modification with $\Delta_{2}=0.5$ (b). Numerical results (solid curves) for $L=10,12, \ldots, 18$ are shown in a log-log plot. In addition to the short-time behavior (dashed curves), the long-time behavior is extrapolated in (b) using the observed exponential scaling with $L$ (dotted curve). Inset: The contribution $I_{4}(t)$ by itself increases with $L$.

where $S_{r}^{x, y, z}$ are the components of spin- $1 / 2$ operators at site $r, L$ is the number of sites arranged periodically, $L+1 \equiv 1$, and $\Delta$ is the anisotropy. The magnetization current

$$
J=\sum_{r=1}^{L}\left(S_{r}^{x} S_{r+1}^{y}-S_{r}^{y} S_{r+1}^{x}\right)
$$

commutes with $H$ in the non-interacting case $\Delta=0$. In that case (due to $\left\langle J^{2}\right\rangle=L / 8,\left\langle J^{4}\right\rangle=3\left(L^{2}-L\right) / 64$, and $\chi=L / 4)$, one obtains directly $D(t)=t / 2$ and $B(t)=-t^{3} / 16$, which for $\Delta>0$ remains only an approximation at short times, in agreement with Eq. (4) of Ref. 9. Remarkably, at $\Delta=0$ a series expansion of density autocorrelations (Bessel functions [16]) leads also to $q^{2} D(t)$ and $-q^{4} B(t)$ as the leading terms, hence being a convincing consistency check of the present approach.

In the metallic regime, $0<|\Delta|<1$, the magnetization current is still partially conserved such that the two-point correlation $f(t)$ does not decay to zero but remains at a finite Drude weight $D_{\mathrm{m}}=\lim _{t \rightarrow \infty} f(t), 0<D_{\mathrm{m}}<1 / 8$, recently proven by establishing positive lower bounds in the thermodynamic limit [4]. This finite Drude weight implies the linear increase of the diffusion coefficient at long times, $D(t) \propto 4 D_{\mathrm{m}} t$, just as in the case of $\Delta=0$. Factoring the four-point correlation at long times [17], 
one derives the asymptotics of the Burnett coefficient as

$$
B(t) \simeq-4 D_{\mathrm{m}}^{2} t^{3} .
$$

In Fig. 1 1 we demonstrate this result by numerically simulating $B(t)$ for finite length $L=10, \ldots, 18$ using all invariant subspaces (translation, magnetization) and also using 4th order Runge-Kutta integration for generating time order 15] (step size $\delta t=0.02$ ). While Fig. [1 clearly shows for $\Delta=0.5$ a stronger than quadratic increase of $B(t)$ with time, it also is consistent with $B(t) \sim-(0.63)^{2} t^{3} / 16$ at long times, e.g., $63 \%$ of the Drude weight in the case of $\Delta=0$. Remarkably, the exact Drude weight at $\Delta=0.5$ for finite $L=18$ [20] is $63 \%$ [62\%] of the Drude weight at $\Delta=0$, while the lower bound in the thermodynamic limit is $56 \%$ [4].

Eventually, we discuss the regime $\Delta>1$, where Drude weights are expected to vanish in the thermodynamic limit and strong evidence of magnetization diffusion has been found in non-equilibrium bath scenarios on the basis of the Lindblad equation [18, 19]. The diffusion coefficient has been shown to behave as $D(t>3.0 / \Delta) \approx$ $0.88 / \Delta$ at vanishing [5] and finite momentum [9]. We focus on the Burnett coefficient $B(t)$ in this Letter. Figure 2 (a) shows numerical results summarizing an order of a CPU-year of simulations and plotting, for convenience, $|\mathrm{d} B(t) / \mathrm{d} t|$ in a log-log scale. Several comments are in order: First, after the already discussed $t^{3}$ behavior at short times, the Burnett coefficient changes its sign, visible as the zero drop in Fig. 2 (a), and indicates a correction towards an insulator. Second, curves for $L \geq 12$ have converged for times after the zero drop and show at least the tendency to form a plateau at $t \sim 4$ for $L \rightarrow \infty$, then indicating a linearly increasing Burnett coefficient $B(t) \propto t$. And third, even though a possible plateau is not pronounced yet, the contributions $I_{4}(t)$ and $I_{2-2}(t)$ increase linearly with time at $t \sim 4$, see the inset of Fig. 2 (a). Notably, the Burnett coefficient turns out to be the small difference between both contributions, which by themselves diverge with $L$. This divergence does not appear in the modular quantum system, discussed later. Finally, we show in Fig. 2 (b) numerical results for the modified non-integrable $X X Z$ model $H+\Delta_{2} \sum_{r} S_{r}^{z} S_{r+2}^{z}$ with $\Delta_{2}=0.5$. While the overall structure of $|\mathrm{d} B(t) / \mathrm{d} t|$ is similar, an emerging plateau is more clearly visible for the accessible lengths, hence pointing towards a linearly increasing Burnett coefficient again. Summarizing, a linear asymptotic scaling $B(t) \propto t$ is clearly suggested in either integrable or non-integrable regimes with a finite diffusion constant.

Heisenberg chains in the large-spin limit.- In addition we present results on the classical limit of the considered spin chains, where we focus on the case $\Delta=1.5$ and $\Delta_{2}=0.5$ only. In that limit the magnetization current is a function of classical unit (angular momentum) vectors. Their dynamics we obtain by numerically integrating the corresponding Hamiltonian equations of motion.
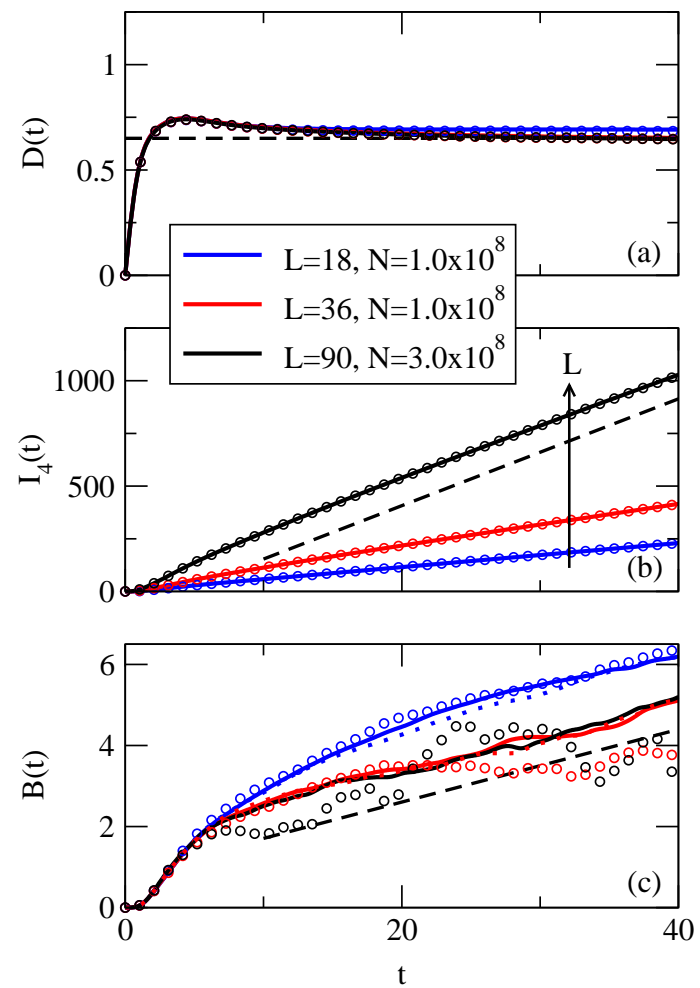

FIG. 3. (color online) Classical (a) diffusion coefficient $D(t)$, (b) the contribution $I_{4}(t)$, and (c) Burnett coefficient $B(t)$ for the $X X Z$ model at $\Delta=1.5$ with $\Delta_{2}=0.5$. Numerical results (solid curves) on finite length $L=18,36,90$ are obtained by numerically integrating the Hamiltonian equations of motion (fixed step size $\delta t=0.05$ ) and by averaging over $N \sim 10^{8}$ completely random initial states. The average over only $N=$ $10^{7} \ll 10^{8}$ is also indicated (symbols). In (c) results for a $2.5 \times$ smaller time step are shown (dotted curves). Straight lines (dashed curves) serve as guides to the eye.

Formally, the diffusion coefficient in Eq. (4) and the Burnett coefficient in Eq. (5) remain defined the same way, but the equilibrium average at high temperatures is now performed by sampling over a set of completely random initial configurations, see Ref. [20] for instance. While the required number of initial states decreases with the chain length $L$ for the evaluation of the diffusion coefficient 21], the situation turns out to be different for the evaluation of the Burnett coefficient. As in the quantum case, the Burnett coefficient is the small difference between two contributions, which by themselves diverge with $L$. Thus, errors due to insufficient averaging increase with $L$ and can only be compensated by taking into account more and more initial configurations. Approximately $N \approx 10^{8}$ initial states are already required for a chain of length $L=90$ taking about a CPU-year of computation time. In Fig. 3 we summarize these results for three different sizes $L=18,36,90$. Apparently, $D(t \gtrsim 10)$ becomes independent of time for all considered lengths $L \geq 18$. Remarkably, the quantitative value $D \approx 0.65$ is close to the expectation for the diffusion con- 

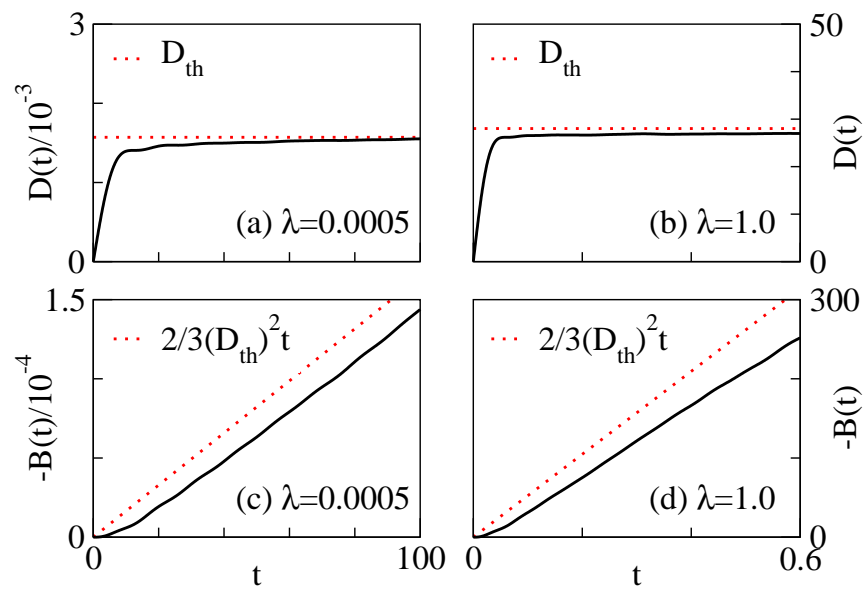

FIG. 4. (color online) Diffusion coefficient $D(t)$ and Burnett coefficient $B(t)$ for the modular quantum system at coupling (a),(c) $\lambda=0.0005$ and (b),(d) $\lambda=1.0$. (Other parameters: $n=500, \delta \epsilon=0.5$.) Numerical results (solid curves) on arbitrary length $L$ are shown for a representative translation subspace (momentum $k=\pi / 5$ ). Results agree well with the theoretical predictions (non-solid curves).

stant in the quantum case 21]. Furthermore, $B(t \gtrsim 10)$ is observed to increase linearly in time, at least for the largest two sizes $L \geq 36$. The latter observation indicates the linear divergence of the Burnett coefficient in diffusive classical spin chains. This is in clear agreement with the finite-size results in the quantum case, see Fig. 2 indicating that the underlying mechanism "survives" the transition to the classical limit.

"Modular quantum system".- Due to a delicate counterbalance of the terms $I_{4}$ and $I_{2-2}$, the calculation of Burnett coefficients for many-body systems is extremely demanding. Thus, in order to corroborate our prediction that $B(t) \propto t$ for one-dimensional lattice systems with finite diffusion constants, we make another numerical experiment in a single-particle diffusive quantum system - the so-called modular quantum system [5, 22]. Each of the $L$ local modules features an identical spectrum, consisting of $n$ equidistant levels in a band with the width $\delta \epsilon$. Therefore the local Hamiltonian at the position $r$ is given by $h_{r}=\sum_{\mu} \mu \delta \epsilon / n|r, \mu\rangle\langle r, \mu|$ in the one-particle basis $|r, \mu\rangle$. The nearest-neighbor interaction between two local modules at the positions $r$ and $r+1$ is $v_{r}=\lambda m_{r}+$ H.c.,

$$
m_{r}=\sum_{\mu, \nu} c_{\mu, \nu}|r, \mu\rangle\langle r+1, \nu|
$$

with the overall coupling strength $\lambda$. The $r$-independent coefficients $c_{\mu, \nu}$ are a single realization of complex, random, and uncorrelated numbers: their real and imaginary part are both chosen corresponding to a Gaussian distribution with the mean 0 and the variance $1 / 2$. Of particular interest is the probability for finding the particle somewhere within the $r$ th local module. The as- sociated local current is $j_{r}=\imath \lambda m_{r}+$ H.c. with a form very similar to $v_{r}$, e.g., almost completely random (apart from the translation invariance and the necessary Hermitian property).

The modular quantum system is one of the few quantum models which have been reliably shown to exhibit diffusion with a finite diffusion constant, reading $D_{\mathrm{th}}^{\mathrm{w}}(t>\pi / \delta \epsilon)=2 \pi \lambda^{2} n / \delta \epsilon$ for weak coupling [22] and $D_{\text {th }}^{\mathrm{s}}=(t>1 / \lambda \sqrt{2 n})=\lambda \sqrt{\pi n / 2}$ for strong coupling [5]. One might expect a finite Burnett coefficient due to both the presence of diffusion and the random elements of the current. For instance, because $J^{2}$ is close to an identity matrix, one may be tempted to factorize as $\left\langle J(0) J\left(t_{1}\right) J\left(t_{2}\right) J\left(t_{3}\right)\right\rangle=f\left(t_{3}\right) f\left(t_{1}-t_{2}\right)$, then allowing for a finite Burnett coefficient. But the latter factorization already fails when all time arguments are equal. In fact, $\left\langle J^{4}\right\rangle=2 f(0)^{2}=8 \lambda^{4} n^{2}$, resulting from an additional coherent sum over the off-diagonal elements of $J^{2}$. Instead, fulfilling the static property, we may choose the unbiased factorization of $\left\langle J(0) J\left(t_{1}\right) J\left(t_{2}\right) J\left(t_{3}\right)\right\rangle$ into $2 / 3\left[f\left(t_{1}\right) f\left(t_{2}-t_{3}\right)+f\left(t_{2}\right) f\left(t_{1}-t_{3}\right)+f\left(t_{3}\right) f\left(t_{1}-t_{2}\right)\right]$, yielding the relation $I_{4}(t)=2 / 3 I_{2-2}(t)$ between the two contributions to $B(t)$. Therefore, noting that $\chi=1$, this choice leads to the linearly increasing Burnett coefficient

$$
B(t>\tau) \simeq-\frac{2}{3} D_{\mathrm{th}}^{2} t
$$

For verification, we present in Fig. 4numerical results on $D(t)$ and $B(t)$. Because the linear growth of the Hilbert space with $L$ is compensated by translation invariance, the dimension of a momentum $k$-subspace is only set by the level number $n$, chosen as $n=500$ to ensure a sufficient number of states. Since we do not find a significant dependence on $k$, Fig. 4 depicts numerical results for a single $k$-subspace. The quantitative agreement with the theoretical predictions on $D(t)$ and $B(t)$ clearly demonstrates a linearly increasing Burnett coefficient despite the existence of a diffusion constant, which is in agreement with the previous results on spin chains, but much clearer due to computational simplicity of the model.

Conclusion.- In this Letter we presented extensive numerical and theoretical investigations of Burnett coefficients in quantum chains. We conjectured and supported the general statement that in the thermodynamic limit Burnett coefficients diverge linearly, $B(t) \propto t$, in diffusive regimes with finite diffusion constants. Recall that this linear divergence is still consistent with diffusion but causes Burnett coefficients to be a relevant correction at arbitrary small momentum. In ballistic regimes with positive Drude weights, on the other hand, we demonstrated the cubic divergence $B(t) \propto t^{3}$. This behavior is remarkably different than for Lorentz billiard type classical systems and and calls for a deeper theoretical analysis.

Financial support by the grant P1-0044 of Slovenian research agency is gratefully acknowledged. 
[1] P. Gaspard, Chaos, Scattering and Statistical Mechanics (Cambridge University Press, 1998).

[2] F. Bonetto, J. Lebowitz, and L. Rey-Bellet, Mathematical Physics 2000 (World Scientific Publishing Company, 2000), Sect. Fourier's Law: A Challenge to Theorists.

[3] R. Kubo, M. Toda, and N. Hashitsume, Statistical Physics II. Nonequilibrium Statistical Mechanics (Springer, 1991).

[4] T. Prosen, Phys. Rev. Lett. 106, 217206 (2011); E. Ilievski and T. Prosen, Comm. Math. Phys (2012), DOI: 10.1007/s00220-012-1599-4.

[5] R. Steinigeweg, Phys. Rev. E 84, 011136 (2011).

[6] N. Hlubek et al., Phys. Rev. B 81, 020405(R) (2010).

[7] M. A. Cazalilla and M. Rigol, New J. Phys. 12, 055006 (2010), and references therein.

[8] J. Sirker, R. G. Pereira, and I. Affleck, Phys. Rev. Lett. 103, 216602 (2009).

[9] R. Steinigeweg and W. Brenig, Phys. Rev. Lett. 107, 250602 (2011).

[10] M. H. Ernst and H. van Beijeren, J. Stat. Phys. 26, 1 (1981).

[11] H. van Beijeren, Rev. Mod. Phys. 54, 195 (1982).

[12] N. I. Chernov and C. P. Dettmann, Physica A 279, 37
(2000).

[13] C. P. Lowe, D. Frenkel, and M. A. van der Hoef, J. Stat. Phys. 87, 1229 (1997).

[14] We define the "static susceptibility" as the square of the conserved quantity [5], e.g. $\chi=\left\langle\left(S^{z}\right)^{2}\right\rangle$ for spin transport with $S^{z}$ being the conserved magnetization.

[15] The time order of $I_{4}(t)$ can be determined by numerically solving a set of coupled differential equations for auxiliary operators $M_{i}(t)$,

$$
I_{4}(t)=\frac{2}{\chi}\left\langle J(0) M_{3}(t)\right\rangle, \quad \dot{M}_{i}(t)=J(t) M_{i-1}(t),
$$

where $M_{0}(t)=1$. Here, we use full exact diagonalization of $H$ to evaluate $J(t)$ in the Heisenberg picture and a 4 th order Runge-Kutta integration.

[16] K. Fabricius, U. Löw, and J. Stolze, Phys. Rev. B 55, 5833 (1997).

[17] T. Prosen, Phys. Rev. E 65, 036208 (2002).

[18] M. Michel et al., Phys. Rev. B 77, 104303 (2008).

[19] T. Prosen and M. Žnidarič, J. Stat. Mech. 2009, P02035 (2009); M. Žnidarič, Phys. Rev. Lett. 106, 220601 (2011).

[20] G. Müller, Phys. Rev. Lett. 60, 2785 (1988).

[21] R. Steinigeweg, EPL 97, 67001 (2012).

[22] R. Steinigeweg, H.-P. Breuer, and J. Gemmer, Phys. Rev. Lett. 99, 150601 (2007). 